\title{
THE PATHOLOGICAL ANATOMY OF DEFICIENCIES BETWEEN THE AORTIC ROOT AND THE HEART, INCLUDING AORTIC SINUS ANEURYSMS
}

BY

\author{
JESSE E. EDWARDS AND HOWARD B. BURCHELL \\ From the Sections of Pathologic Anatomy and of Medicine, Mayo Clinic and Mayo Foundation, Rochester, \\ Minnesota, U.S.A.
}

(RECEIVED FOR PUBLICATION MARCH 7, 1957)

A ruptured aneurysm of an aortic sinus (Valsalva) represents a serious threat to the patient's life, chiefly in its tendency to cause cardiac failure. When this lesion occurs alone the clinical diagnosis is relatively simple to make. Particularly is this true should cardiac catheterization and perhaps aortographic studies be included in the investigation of the patient. When a ruptured aneurysm of an aortic sinus occurs in association with a second lesion, particularly ventricular septal defect, the presence of the latter lesion may not be readily established even with the aid of the special studies named.

Surgical therapy for an aneurysm of an aortic sinus has only recently been introduced. In view of this, it is essential that the anatomical relations of the aortic sinuses and of aneurysms occurring in these regions be understood. At the same time, it is important to appreciate the nature of lesions that may be associated with aortic sinus aneurysm.

In study of the nature of aortic sinus aneurysms we observed that the essential lesion is a separation between the aortic media and the heart (Edwards, Burchell, and Christensen, 1956). This separation or lack of fusion, occurs at the annulus fibrosus of the aortic valve and consequently is located above the valve. It is also apparent that there are cases in which the separation between the aortic media and the heart is below the aortic valve. These lesions, which may occur independently of aortic sinus aneurysm, produce different gross anatomical lesions from those defects that occur above the aortic valve. They have in common with defects above the aortic valve the feature that the nature of the resulting lesion depends only in part on whether it lies above or below the valve, but also to which of the three of the aortic sinuses or valve cusps it is related.

The purpose of this communication is to review the anatomy of the aortic sinuses and the patho- logical anatomy of those lesions which lie at the aortic root and represent a deficiency between the aortic media and the heart. Deficiencies both above and below the aortic valve will be covered.

The anatomy of the aortic root has already been reviewed. Gross and Kugel (1931) emphasized the structure of the aortic valve leaflets and presented some details regarding the relations of the aortic sinuses to surrounding structures.

Ostrum, Robinson, Nichols, and Widmann (1938) emphasized the anatomical features as they pertain to radiological interpretation. In describing the anatomy of the aortic sinuses, it is our purpose to emphasize those relations which are of importance for the orientation of the surgeon.

It is not uncommon that as a result of infection, chiefly bacterial endocarditis and less commonly syphilis, deficiencies in the areas of the aortic sinuses may develop. We are not chiefly concerned in this paper with lesions of a primarily inflammatory nature, although the anatomical relations of deficiencies so developing have similarities to those which occur spontaneously and with which we are here concerned.

Emphasis in this paper will be on pathological material which we ourselves have examined. Comprehensive reviews on the subject of aortic sinus aneurysm have been presented by Raman and Menon (1919), by Jones and Langley (1949), and by Oram and East (1955).

\section{Anatomy of THE AORTiC SinUSes}

Normally, there are three sinuses at the aortic valve, each related to one of the three cusps of the valve. The sinus is a specialized part of the aortic lumen, being walled by its respective valve cusp medially and by the origin of the aorta laterally. The coronary arterial ostia are related to the aortic sinuses, but since these normally arise at a level just 
distal to the level of the upper edge of the valve cusps, they do not, in the strict sense, arise from the aortic sinuses but rather from the aortic wall just above the sinuses.

The names given to the aortic sinuses are the same as those for the valve cusps to which each is related. In this paper we shall speak of left, right, and posterior aortic cusps and sinuses. The left and right sinuses are the ones above which the left and right coronary arteries respectively arise. The posterior sinus (sometimes called the non-coronary) is the one not directly related to a coronary arterial ostium.

In the body the aortic valve is so orientated that the stream of blood flowing through it is directed to the right and upward. The valve does not lie in the same transverse plane of the body. The portion of the left aortic cusp nearest the right is most superior and anterior, while the posterior cusp and the adjacent part of the left cusp lie at the most inferior and posterior levels. The most anterosuperior portion of the left aortic cusp lies at the same level as the nearby left cusp of the pulmonary valve. Otherwise, the aortic valve lies inferior to the pulmonary valve. The inferior position of the posterior part of the aortic valve may be appreciated from the fact that, at this level, the aortic valve lies inferior to the transverse sinus of the pericardium and anterior to the adjacent portions of the two atria and atrial septum.

Fig. 1 illustrates a gross section through the normal heart, aorta, and pulmonary trunk at a level above the two semilunar valves. The plane

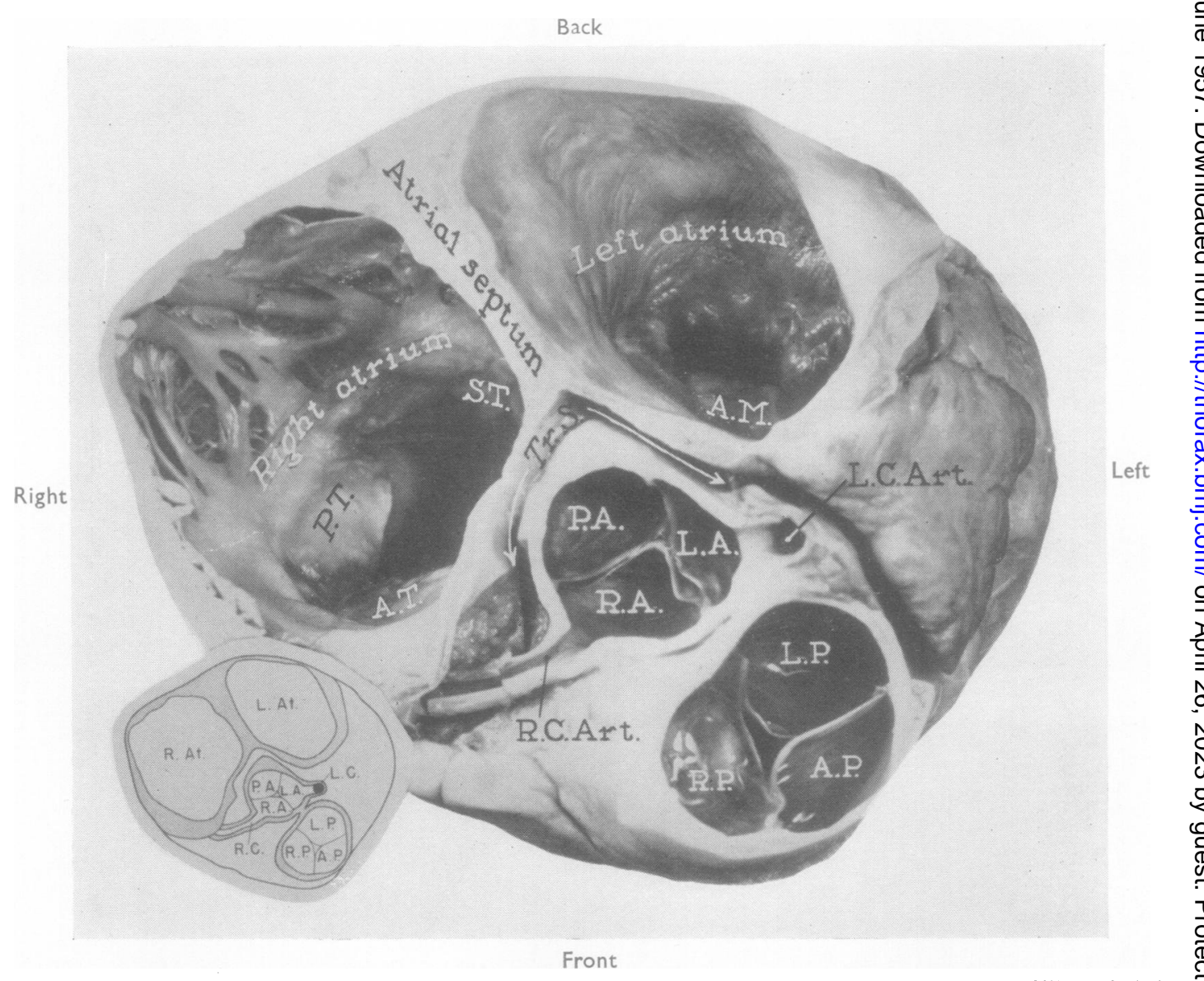

FrG. 1.-Gross section through base of normal heart. The plane of section is more superior anteriorly (lower portion of illustration) than posteriorly (upper portion of illustration). In the inset is a simplified diagram of the photograph to assist in understanding the planes of sections used in subsequent illustrations. R.P., L.P., and A.P. =right, left, and anterior pulmonary cusps and sinuses, respectively. R.A., P.A., and L.A. = right, posterior, and left aortic cusps and sinuses, respectively. R.C. and L.C. $=$ right and left coronary arteries, respectively. Tr.S. $=$ transverse sinus of pericardium. In right atrium, A.T., P.T., and S.T. $=$ anterior, posterior, and septal leaflets, $O$ respectively, of tricuspid valve. In left atrium, A.M.= anterior mitral leaflet. The posterior leaflet of the mitral valve is shown opposite the anterior leaflet and below the word " atrium." 
of the section is such that at the front of the specimen it corresponds to a more superior level in the body than at the posterior part of the specimen. In the illustration an inset appears which in a simplified way shows the essentials of the photograph appearing in the same figure. The simplified diagram will appear in subsequent illustrations with planes of the section indicated of normal aortic sinuses that are shown in low-power photomicrographs.

The Right Aortic Sinus.-Not only does each aortic sinus have relations that are quite different from those of the other two sinuses but, significantly, differences in relationships exist at different parts of each sinus. Ostrum and his associates (1938) divided each sinus into two parts. The differences are perhaps best understood if each aortic sinus is considered as having three more or less equal parts as follows: (a) a central or intermediate third and $(b$ and $c$ ) two other thirds, each adjacent to one of the other two valve cusps and sinuses. Thus the right aortic sinus and cusp may be said to have an intermediate part, a posterior part which lies next to the posterior aortic cusp and sinus, and a left part lying next to the left aortic cusp and sinus.

The right aortic sinus is entirely related to a cardiac chamber, mainly, the right ventricular outflow tract. The central part of the sinus lies against the crista supraventricularis, the prominent ridge seen in the outflow tract of the right ventricle in the upward prolongation of the muscular part of the ventricular septum (Fig. 2). The left part of the right aortic sinus is the most anteriorly located of the three parts of this sinus. It abuts against the outflow tract of the right ventricle in the angle between the upper aspect of the crista supraventricularis and the pulmonary valve. In cases in which the crista is particularly large, a portion of it may be related to part of the left portion of the right aortic sinus.

The posterior third of the right aortic sinus, that third that lies next to the posterior aortic sinus, lies mainly against the right ventricle just posteroinferior to the crista supraventricularis. The most posterior aspect of this third of the sinus may lie against the right atrium at the base of the septal leaflet of the tricuspid valve.

Below all parts of the right aortic sinus lies the ventricular septum. Beneath the central and left parts the muscular portion is constant. Beneath the posterior part of the right aortic sinus lies either the muscular or the membranous portion of the ventricular septum, depending on the size of the latter. When the membranous portion is large, it lies in relation to the posterior part of the right sinus.
Otherwise, and more commonly, the muscular portion is in this position.

The POSTERIOR AORTIC SINUS.-The posterior aortic sinus, like the right, is also related entirely to intracardial structures, which are the right and left atria and the atrial septum. The posterior aortic cusp and sinus may also be divided into three parts, which are the central, the right, and the left.

Adjacent to the right and intermediate parts of the posterior aortic sinus is the atrial septum (Fig. 3). Lesions of this region are directed into the right or left atrial cavities or directly into the atrial septum, depending on the extent and direction of the process.

The inferior relations of the right part of the posterior aortic sinus are similar to those of the adjacent part of the right aortic sinus. Here the ventricular septum lies, either the muscular or the membranous portion, again depending on the size of the membranous septum. Beneath the central or intermediate portion of the posterior aortic sinus the membranous portion of the ventricular septum is a constant structure.

The left part of the posterior aortic cusp shares with the adjacent third of the left aortic leaflet the peculiarity of being continuous with tissue of the anterior mitral leaflet inferiorly (Figs. $3 a$ and $4 a$ ). Here is the only part of the aortic wall which is not connected with the ventricular portion of the heart; rather, the aorta is connected with the base of the anterior mitral leaflet. Adjacent to the left part of the posterior aortic sinus lies the left atrial wall.

The Left Aortic Sinus.-The left aortic sinus and cusp may also be divided into three parts for descriptive purposes. These are the central or intermediate, the posterior and the right.

The posterior part of the left aortic cusp and sinus is adjacent to the posterior cusp and sinus. Its relations are identical to those of the adjacent third of the posterior sinus and cusp (Figs. $3 a$ and $4 a)$.

The other extreme of the left aortic cusp and sinus is termed the right part and lies in an anterior position and adjacent to the right aortic cusp and sinus. Since this third of the left cusp is the only one related to the ventricular septum, it may also be called " the septal part of the left aortic cusp."

This part of the left aortic cusp and the corresponding wall of the aorta are connected inferiorly with the most anterosuperior aspect of the muscular part of the ventricular septum (Fig. 4b). This is the portion of the aortic valve that lies most superiorly in the body. Adjacent to the right part of the left aortic sinus is the wall of the pulmonary trunk at the level of the left pulmonary sinus. 


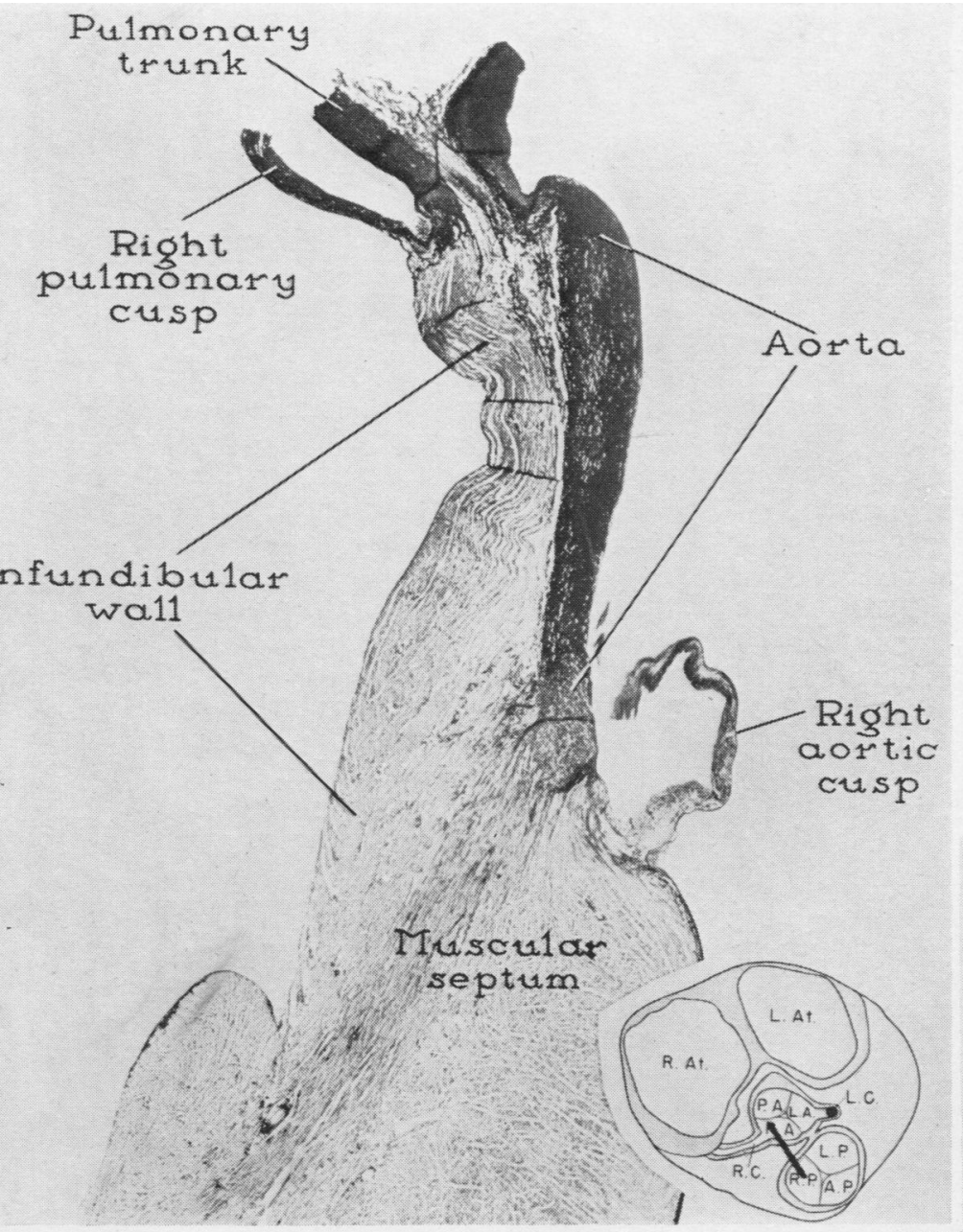

Fig. 2a

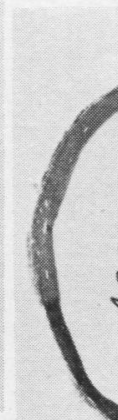

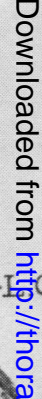

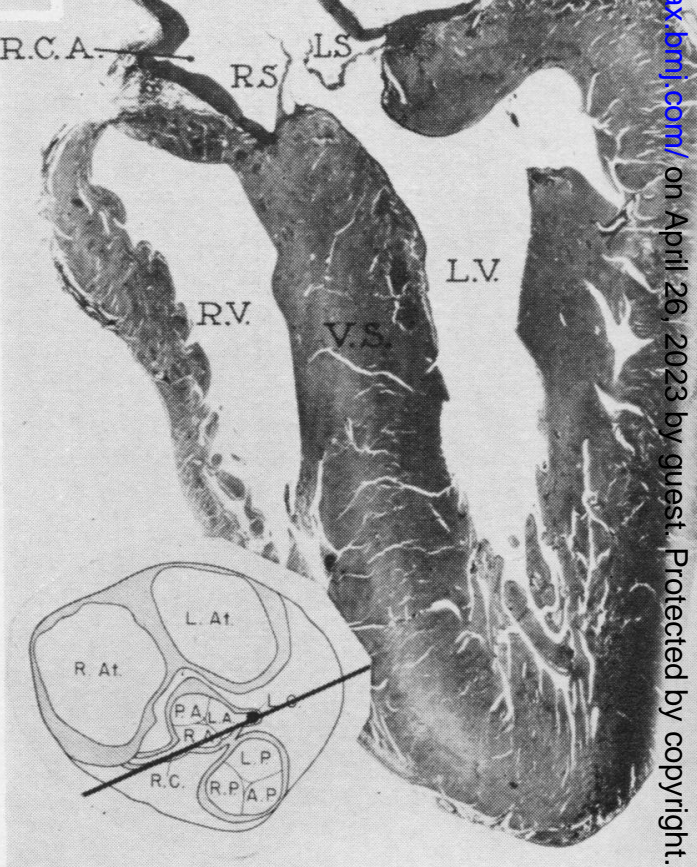

FIG. 2a.-Low-power "photomicrograph of longitudinal section through normal central third of right aortic cusp and sinus and through right pulmonary cusp. Plane of section indicated by heavy line in inset. Beside this portion of the aortic valve lies the upper prolongation of the ventricular septum (infundibular wall), which forms a part of the wall of the outflow tract of the right ventricle and which includes the crista supraventricularis. Below this portion of the right cusp and sinus lies the muscular portion of the ventricular septum. Note the normal distance between right pulmonary cusp and central part of right aortic cusp. Note also intimate connexion between aortic media and origin of aortic cusp (annulus fibrosus). b.-Low-power photomicrograph through entire normal heart sectioned through central portions of the right and left aortic cusps and sinuses. The pulmonary trunk lies anterior to this plane of section and is not shown in the illustration. The relations of the right aortic sinus (R.S.) are essentially the same as illustrated in $a$. The relations of the left aortic sinus at its central portion (L.S.) are of especial interest in that this is the only part of the aortic origin that does not lie against a cardiac chamber or pulmonary trunk, but instead lies against the epicardium. 


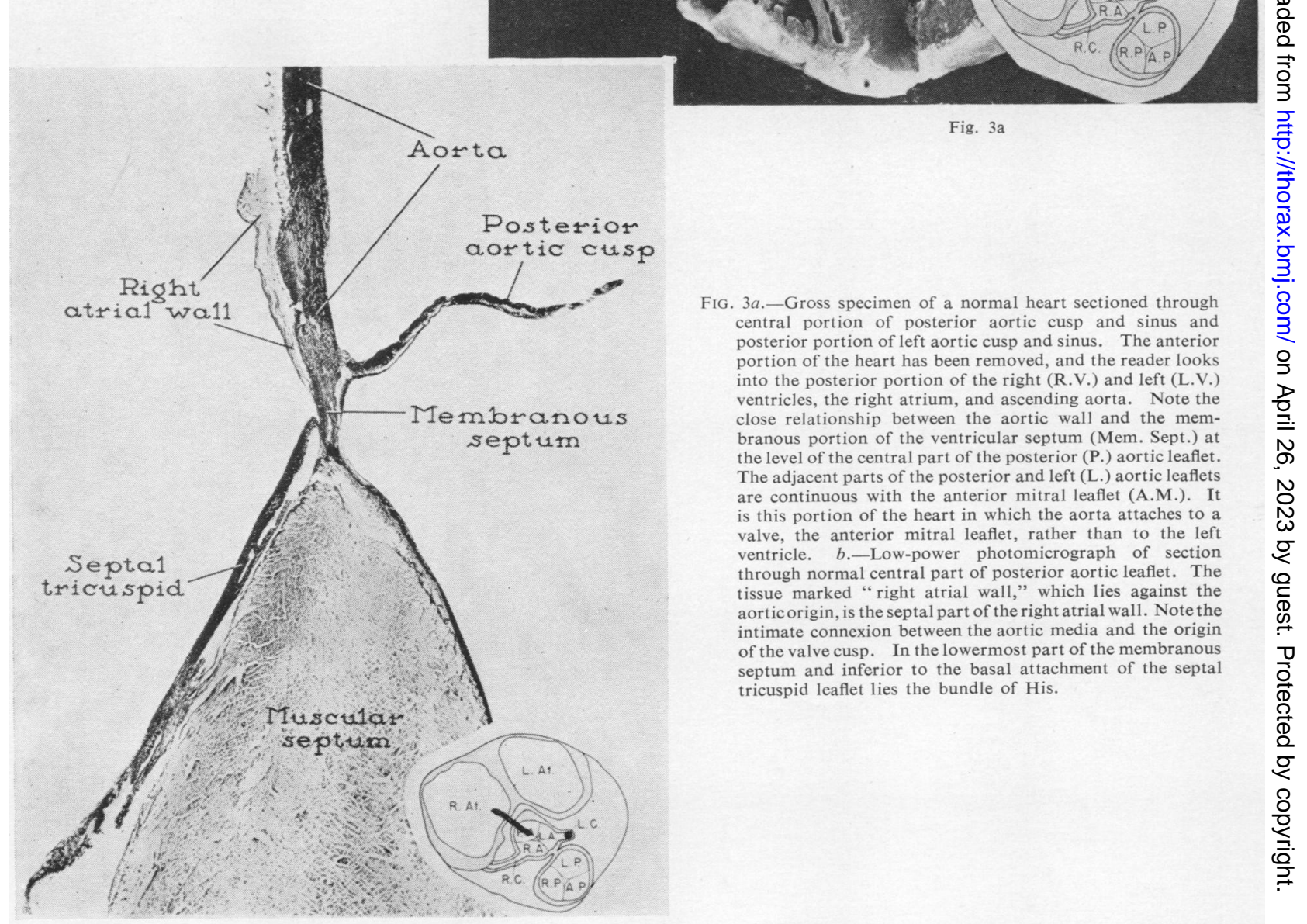




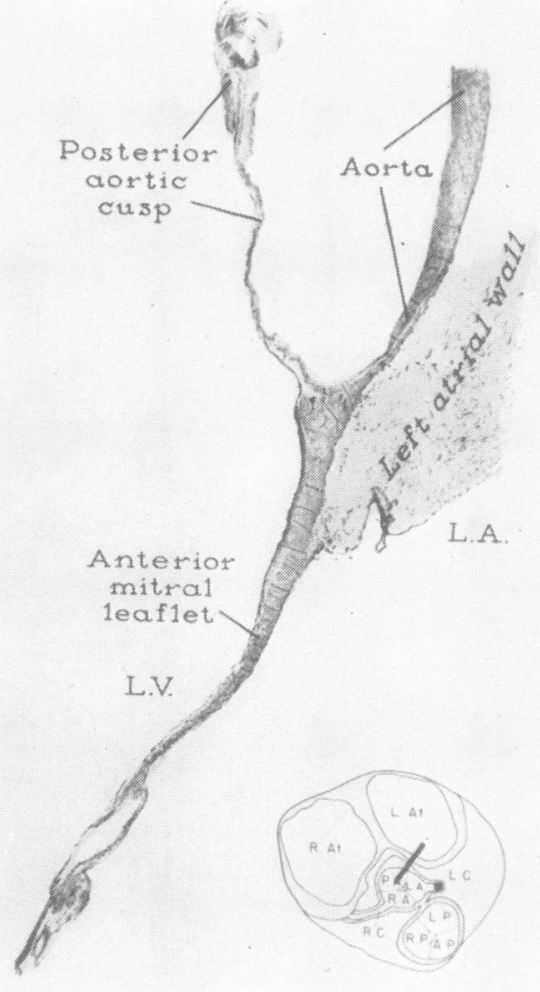

Fig. $4 a$
FIG. 4a.-Low-power photomicrograph through the normal left third of the posterior aortic leaflet. There are striking differences in relations at this level as contrasted with those of the central third of the posterior aortic cusp and sinus which are illustrated in Fig. $3 b$. For gross illustration of region through which this section was taken, see Fig. $3 a$. The lower attachment of the a anterior mitral leaflet. Here and in relation to the adjacent third anterior mitral leaflet. Here and in relation to the adjacent third
of the left aortic cusp and sinus, the aorta does not connect with the left ventricle, but rather with the anterior mitral leaflet. b.-Low-power photomicrograph through normal right (septal) third of left aortic leaflet and adjacent left pulmonary leaflet. This portion of the aortic valve lies most anteriorly in the body. Inferior to the cusp and sinus is the muscular ventricular septum, and adjacent to it is the origin of the pulmonary trunk at the level of the left pulmonary leaflet and sinus. The orientation of the aortic valve differs from that of the pulmonary valve, hence the difference in planes of section through the two cusps. The pulmonary cusp is cut longitudinally, while the aortic cusp is cut obliquely and almost horizontally.

The third part of the left aortic cusp and sinus lies between the two afore-mentioned ones and is the intermediate or central part. The intermediate portion of the left aortic cusp and sinus has the peculiarity of being the only region of the aortic root that is not adjacent to a cardiac chamber or the pulmonary trunk. This part of the left cusp and the corresponding part of the aortic wall are connected with the lateral aspect of the left ventricular base. Adjacent to the wall of the sinus in this region is the epicardium. It is above this part of the left aortic sinus that the left coronary artery arises. 


\section{Defects Between the Aortic RoOt AND THE HeART OCCURRING Singly}

Defects Above the Right Aortic CuSP.-In our collection, there are three cases which portray a defect between the aortic root above the level of the right cusp and the heart, each occurring in men whose ages ranged from 63 to 75 years. In each case the lesion was found incidentally at necropsy. In two of the instances, the defect was not associated with aneurysm formation. The base of the defect was walled by the crista supraventricularis of the right ventricle, which could be seen from the aortic lumen (Figs. $5 a$ and $b$ ).

In the third case, at the site of the deficiency, there was an aneurysm of the right aortic sinus, beyond which the crista supraventricularis bulged into the right ventricle (Fig. 6). In none of the three cases cited had a rupture occurred, although it is recognized that such complications occur in aneurysms of the right aortic sinus. When this happens, the rupture of the aorta is almost always into the outflow tract of the right ventricle.

In an additional case, we observed a ruptured aneurysm of the right aortic sinus, but in this instance there was in addition a ventricular septal defect. The case will be discussed in the later section dealing with combinations of defects between the aortic root and the heart.

In an unusual instance an aneurysm may involve the most posterior portion of the right aortic cusp and so may rupture into the right atrium, into the right ventricle, or into both (Oram and East, 1955).

Warthen's (1949) case of ruptured aneurysm of the right aortic sinus was unusual in that the aneurysm burrowed downward in the ventricular septum and then communicated with the left ventricle. A clinical picture resembling that of aortic valvular insufficiency resulted.

In the case of Raman and Menon (1949) the aneurysm originating in the right aortic sinus presented into both the right and left ventricles but had not ruptured into either.

Defects Below the Right AORTIC CusP.Defects of continuity between the aorta and the heart that occur below the right aortic cusp appear as ventricular septal defects. Usually the deficiency is not restricted to the area beneath the right cusp but also includes varying amounts of deficiency beneath an adjacent aortic cusp. When there is a defect beneath the right aortic cusp and an adjacent cusp, the posterior cusp is usually related to the defect, less commonly the left cusp. In the commonest situation the defect lies beneath the intermediate and right parts of the posterior cusp and beneath the posterior part of the right cusp.
Fig. 5-From a man, 68 years old, with separation of the aortic media from the heart at the right aortic sinus, but without aneurysm formation. a.-Gross photograph of aortic valve. The aneurysm formation. a.-Gross photograph of aortic valve. The portion of the right aortic sinus (R.), allowing the crista supraventricularis of the right ventricle to be seen from this view. $\mathbf{L}=$ left and $\mathbf{P}$.=posterior aortic cusps. b.-Low-power photomicrograph through central portion of right aortic sinus. cated from the heart, but no aneurysm has yet formed. The crista supraventricularis (C.S.) forms the wall of the right aortic sinus.

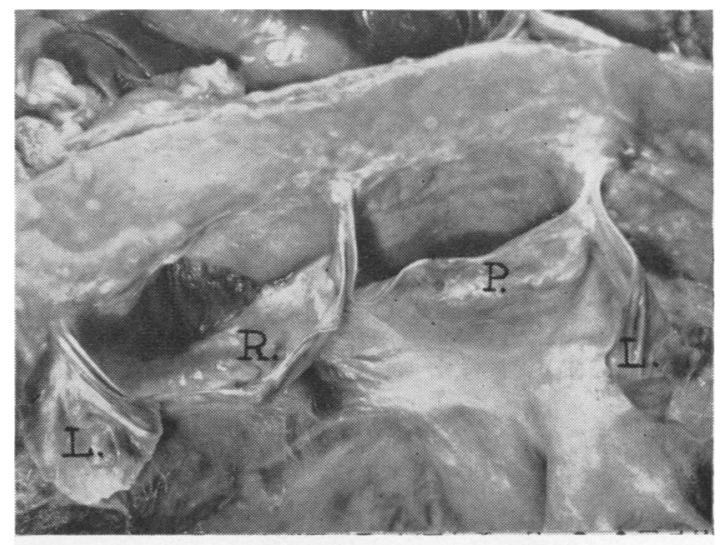

Fig. 5a

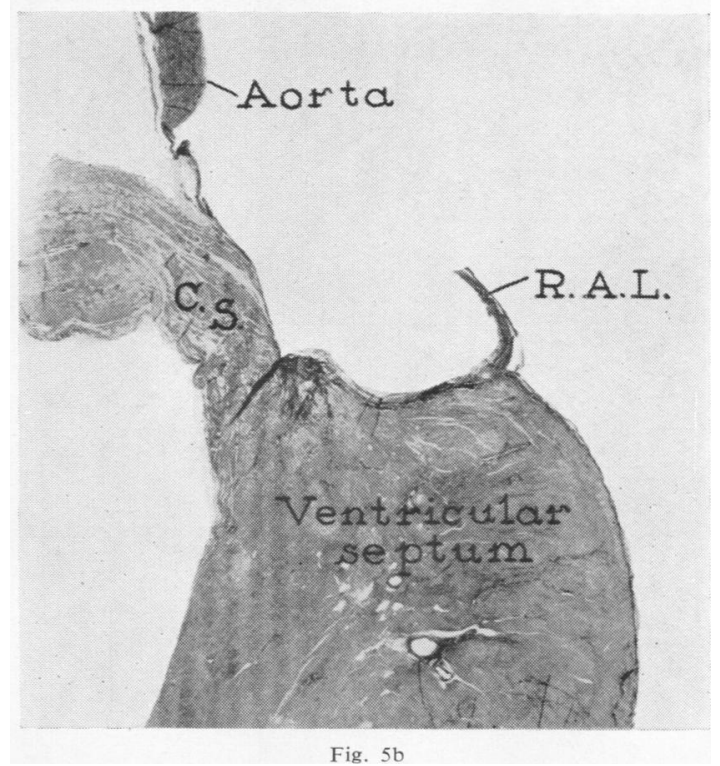

These defects represent the commonest type of ventricular septal defect. From the right ventricular aspect they lie in the right ventricular outflow tract between the crista supraventricularis above and the area where the membranous septum lies, or should lie, below. Often the membranous septum is absent, in which case the postero-inferior wall of the defect is formed by the anterior surface of the 


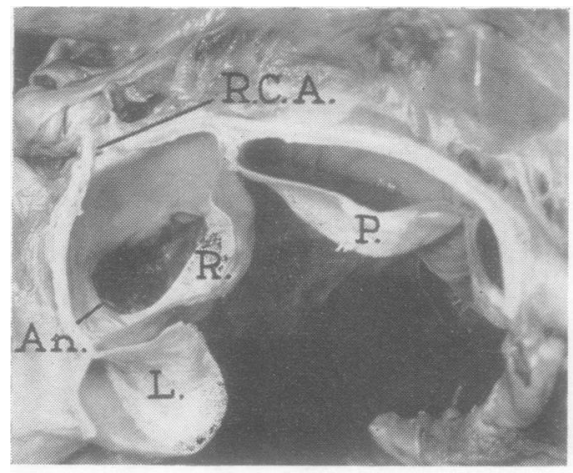

Fig. 6a

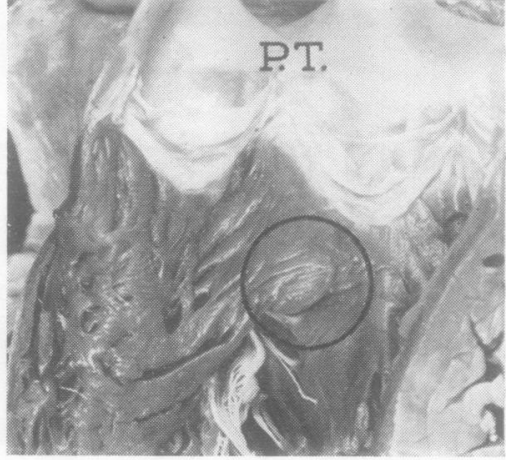

Fig. $6 \mathrm{~b}$

FIG. 6.-From a 75-year-old man with an unruptured aneurysm of the right aortic sinus. $a$-At the right aortic sinus there is an aneurysm, the?floor of which is formed by the crista supraventricularis of the right ventricle. $b$. -The outflow tract of the right ventricle and the $\sigma$ pulmonary valve. Within the circle is an unruptured aneurysm at the crista supraventricularis. c.-Low-power photomicrograph of the $\Omega$ central portion of the right aortic sinus, showing separation of the aortic media from the heart. The aneurysm of the aortic sinus is walled 0 by atrophic muscular tissue, which is the crista supraventricularis of the right ventricle. 
anterior mitral leaflet where it joins the septal leaflet of the tricuspid valve. The anatomical details of this and of the other types of ventricular septal defect have been described in another paper from this institution (Becu, Fontana, DuShane, Kirklin, Burchell, and Edwards, 1956).

When a defect lies inferior to the left third of the right aortic cusp it usually also includes the tissue beneath the adjacent (right) third of the left aortic cusp. These defects form the less common variety of ventricular septal defects in the outflow portion of the ventricular septum. From the right side they all are seen to lie just inferior to the right and left pulmonary cusps and above the crista supraventricularis.

Defects Above the Posterior Aortic Cusp.When lack of continuity exists between the aortic media and the heart above the posterior aortic cusp, the defect is usually in relation to the intermediate part of the sinus. The lesion represents the classic aneurysm of the posterior aortic sinus, which is less common than aneurysm of the right aortic sinus. The basic lesion is the same in either location. The usual aneurysm of the posterior aortic sinus presents in the septal wall of the right atrium, and rupture into this chamber may occur. In the septal wall of the right atrium, the aneurysm lies just superior to the annulus fibrosus of the septal leaflet of the tricuspid valve and in this position is closely related to the membranous portion of the ventricular septum and to the conduction bundle of His. While aneurysms of the right aortic sinus are frequently associated with ventricular septal defect, aneurysms of the posterior aortic sinus are usually not associated with such a defect. An exceptional case in which the two were associated will be described in a subsequent section of this paper.

The one example of an aneurysm of the posterior aortic sinus occurring as a single lesion involved a woman who was 42 years of age at the time of her death from cardiac failure. The essential pathological features of this case have been described in an earlier report (Edwards and others, 1956).

While it is theoretically possible on the basis of anatomical relationships for aneurysms arising from the intermediate part of the posterior aortic sinus to rupture into the left atrium or simply to enter the atrial septum, we do not know of such an occurrence in examples of uninfected aneurysm. In a case of bacterial endocarditis described by Orbison and Mostofi (1956), a mycotic aneurysm developing in this position ruptured into the atrial septum. This caused a haematoma of the atrial septum to form, but there was no communication with either atrial cavity. We are not aware of aneurysms restricted to either the right or the left parts of the posterior aortic sinus, though, theoretically, such lesions could occur. Should this happen, those originating from the right part would present into the right atrium and those from the left part into the left atrium.

Defects Above the Left Aortic Cusp.Aneurysms restricted to the left aortic sinus are rare. Oram and East (1955) have credited Higgins (1934) with reporting the occurrence of an aneurysm of the left aortic sinus which ruptured into the right atrium. Herson and Symons (1946) interpreted Macleod's (1944) case similarly. Review of these two reports makes it doubtful that they were correctly interpreted. The use of different terminology for the aortic sinus from that which we employ makes an exact interpretation impossible. Nevertheless, it is probable that both in Higgins' and in Macleod's respective cases the aneurysms originated in the posterior (non-coronary) aortic sinus.

Dilatation of all three aortic sinuses has been reported pathologically by Micks (1940).

In the case of Raman and Menon (1949) a 29year-old man showed aneurysms of the right and the left aortic sinuses. Neither aneurysm had ruptured. The aneurysm of the left sinus presented in the epicardium medial to the left auricular appendage. Death of the patient seemed related to complete heart block.

No specimen with an aneurysm of the left aortic sinus is in our pathological collection. A specimen which appears to be an example of this was shown to one of us (Edwards) by Dr. John Coe, of Minneapolis. In this case there was an aneurysm centred at the intermediate part of the left aortic sinus, the mouth of the aneurysm lying at the lowermost part of the sinus and therefore relatively distant from the origin of the left coronary artery. The aneurysm presented into the epicardium above the left auricular appendage and behind the aorta. The body of the aneurysm communicated with the right atrial chamber through the anterior wall of the latter. Such a case brings up for consideration the question whether the lesion represents communication of an accessory left coronary artery with the right atrium and aneurysmal dilatation of the accessory artery. The facts that the mouth of the aneurysm lay in the depths of the sinus and that the wall of the aneurysm was shown on histological examination to be composed of non-specific connective tissue rather than arterial wall deter one from accepting such an explanation of the pathogenesis. The same facts suggest that the basic lesion is a separation of the aortic wall from the 
left ventricle at the level of the intermediate part of the left aortic sinus. The aneurysm is then explained as a false aneurysm resulting from an epicardial haematoma which ruptured secondarily into the right atrium.

Defects Below the Left Aortic Cusp.-We are not aware of any examples of separation between the aorta on the one hand and the heart on the other beneath the posterior part of the left aortic cusp. However, examples of separation beneath the intermediate part and beneath the right (septal) part exist.

In another case which was observed at necropsy by Dr. Coe and the specimen kindly sent to us, there was a separation between the aorta and the heart beneath the intermediate part of the left aortic cusp (Fig. 7). This is the area, it will be recalled, in which the aortic valve is not related to a cardiac chamber or to the pulmonary trunk. The lesion in this particular case had the nature of an aneurysm of the base of the left ventricle. We consider that, following separation between the aorta and the heart, there had been a haemorrhage, which was not fatal, into the epicardium. This became organized to create the fibrous and partly calcified wall of the aneurysm. In the strict sense this is a false rather than a true aneurysm of the left ventricle. Two cases which bear some similarity to this one have recently teen reported by Barnard and Brink (1956).

The right or septal part of the left aortic cusp is related to the ventricular septum and the right ventricle. In this position lack of continuity between the aorta and the heart is represented by the relatively uncommon type of ventricular septal defect which on the right side lies above the crista supraventricularis and just below the pulmonary valve. As has been indicated in the section regarding defects below the right aortic cusp, when a defect exists beneath the adjacent parts of either the right or the left aortic cusp, it is usually beneath both.

\section{Combined Defects Between the Aortic Root AND THE HEART}

At times certain lesions which occur singly and which have been described in the previous section may appear in combination with one another in the same heart. It is important to emphasize this, since, when such lesions occur in combination, it may be difficult or impossible by functional studies to determine that two lesions exist rather than one. When two defects occur together, it is usual but not invariable that the loss of continuity between the heart and the aorta is related to the same aortic cusp. Specifically, the combination of lesions usually means that an aneurysm of an aortic sinus: is associated with a ventricular septal defect beneath듬 the corresponding aortic cusp, but there are rare $\frac{\bar{\rho}}{\sigma}$ exceptions wherein a combination of lesions is $\underset{\Omega}{\mathbb{Q}}$ represented by other gross alterations, including aneurysms of two or three aortic sinuses (Raman $\rightarrow$ and Menon, 1949).

Defects A.Bove AND Below THE Right Aortic $\overrightarrow{\vec{\omega}}$ CUsP.-In one case in our collection there was a $\stackrel{\text { }}{\rightleftharpoons}$ combination of defects above and below the right $\vec{x}$ aortic cusp that was represented grossly by a com- $\vec{N}$ bination of aneurysm of the right aortic sinus and a i ventricular septal defect. This case has previously $\vec{N}$ been reported (Morgan and Burchell, 1950; Burchell $\mathrm{G}$ and Edwards, 1951). It seems representative of a number of reported instances wherein these two lesions were combined. Basically, the abnormality in this case was represented by a wide gap between $\Phi$ the lower end of the aorta and the ventricular $\vec{e}$ septum (Fig. 8a). Though, in essence, there was one $Y$ deficiency, there appeared to be two by the presence of the right cusp of the aortic valve. At the involved region, the mid-portion of the right aortic cusp had attachment neither to the aorta nor to the heart. The cusp was maintained in its relatively normal ${ }^{\circ}$ position by the fixation imparted by its lateral $\stackrel{\varrho}{\overrightarrow{7}}$ attachments.

From the right ventricular aspect in this case, the aneurysm lay centred at the expected location of the crista supraventricularis. Because of its large size the upper aspect of the aneurysm was adjacent to $\vec{\sigma}$ the pulmonary valve. Several perforations were present in the aneurysm which allowed a communica- -0 tion above the aortic valve between the aorta and the 3 . right ventricular outflow tract. Below the aortic valve the defect between the aorta and the heart was represented by a ventricular septal defect.

In yet another case, which involved a 21 -year-old woman, there was a peculiar combination of defects $\frac{D}{2}$ which resulted in an abnormal communication between the aorta and the left ventricle in the $N$ presence of a basically normal aortic valve (Fig. $8 b$ ). N In this case there was a separation between the N aorta and the heart, centred at the commissure ${ }_{\sigma}^{\omega}$ between the left and the right aortic cusps. This deficiency was not associated with an interventricular $\frac{0}{\circ}$ communication. Instead, the deficiency led intoof an aneurysm, which lay against the outflow tract of the right ventricle and the origin of the pulmonary trunk.

The aneurysm also communicated with the right $\frac{\rho}{\Phi}$ aortic sinus. In the usual circumstance, when $a \frac{2}{\sigma}$ deficiency exists in an aortic sinus it is at the junction of the aorta and the heart. Here, theo deficiency was somewhat higher in that some aortio 


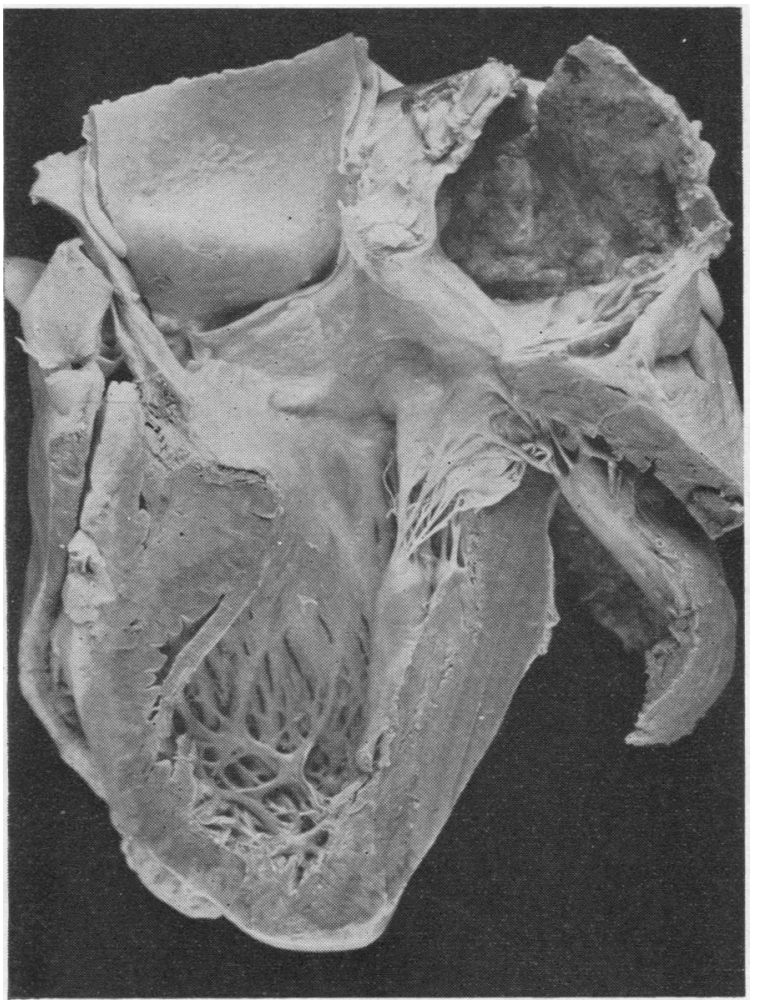

Fig. 7a

FIG. 7.-From a 39-year-old woman with an aneurysm at the base of the left ventricle (case of Dr. J. I. Coe). a.-The left ventricle and aortic origin. The mouth of the aneurysm lies between the aortic and the mitral valve and is centred at the level of the central portion of the left aortic leaflet. The aneurysm protrudes into the epicardium. b.-Close-up view of area involved by aneurysm. The mouth of the aneurysm lies between the central portion of the left aortic leaflet above, and the left ventricle just in front of the mitral valve below. This lesion is assumed to be a separation between the aorta and the heart inferior to the aortic valve and at the level of the central portion of the left aortic leaflet.

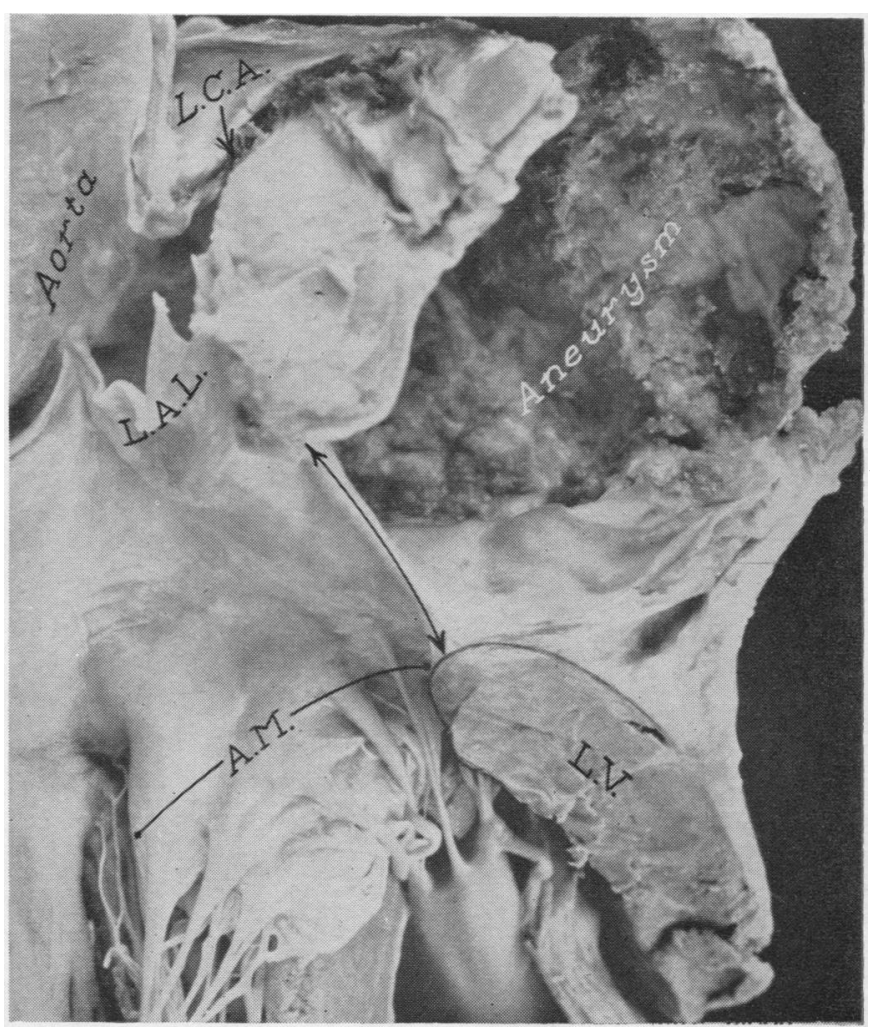

Fig. 7b

tissue remained in the depths of the involved right sinus. Pathogenetically, we consider that the following events had transpired to create the lesions. It is assumed that the first process was a rupture between the aorta and the heart, centred at the commissure between the right and the left cusps. It is further assumed that the rupture between the aorta and the heart was continued into the aorta itself at the right aortic sinus. The two defects then communicated with a haematoma, which became organized to form the (false) aneurysm.

Regurgitation of aortic blood into the left ventricle was accomplished by aortic blood flowing into the aneurysm and from the latter into the left ventricle through the deficiency beneath the aortic valve.

In this case the right coronary ostium was narrow and the lumen was completely obstructed as the vessel passed through the aortic wall. It is considered probable that, at the time of the presumed tear in the aorta, the ostium of the right coronary artery had been torn away and that secondary changes (haematoma or thrombosis) led to the occlusion of the right coronary artery as it passed through the aortic wall. 


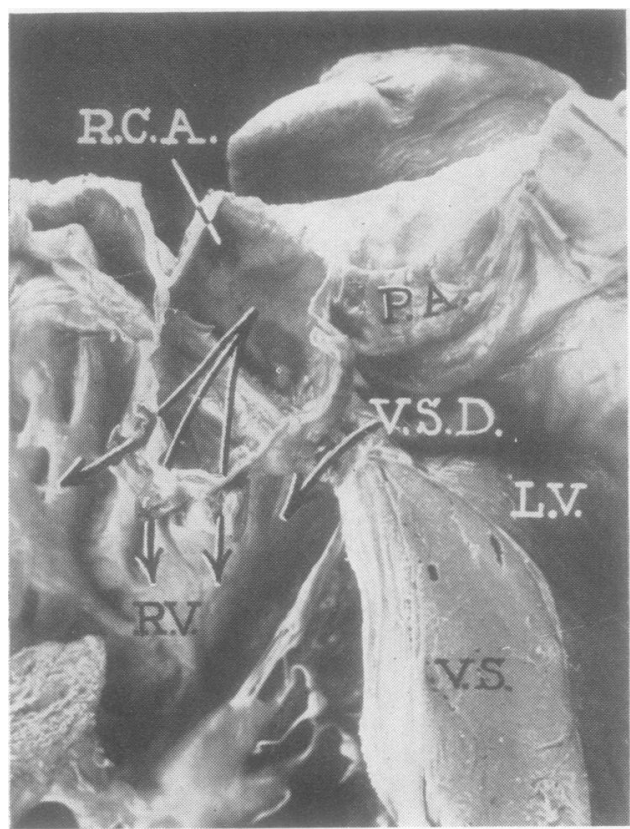

Fig. 8a

FIG. 8a.-From a 36-year-old man. Sagittal section through the outflow tracts of both ventricles and the aortic and pulmonary valves in a case with aneurysm of the right aortic sinus and a ventricular septal defect immediately subjacent to the aneurysm The two lesions are considered to be a separation of the aorta from the heart of extensive nature, and lying both above and below the levels of the right aortic leaflet. P.A.-- posterior aortic cusp. b.-From a 21-year-old woman. The aortices valve and origin of the aorta. There is a separation betweenthe aorta and the heart at the commissure between the left and $\overrightarrow{0}$ the right aortic leaflet. This separation has extended into the aortic wall at the level of the right aortic sinus. Each of these $\overrightarrow{-}$ openings communicates with an aneurysm, through which bloodw above the aortic valve was regurgitated into the left ventricle. Edges of the tear have been indicated by bold lines.

Defects Above Posterior A.ortic CusP and $\vec{N}$ Below Commissure Between Right and Posterior? AORTIC CUSPS.--In the case of a 28-year-old man $\vec{N}$ we found a large aneurysm of the posterior aortico sinus (Fig. 9a) and a ventricular septal defect below? the commissure between the right and the posteriore aortic cusps.

The aneurysm of the aortic sinus resembled the aneurysm of the posterior aortic sinus whicho occurred singly and to which we referred in a fore.. going section. In the case now considered, the defect응 was larger and occupied most of the sinus but was centred at the intermediate part of it. The aneurysm, which presented into the right atrium through the septal wall of the latter, showed several perforations $\stackrel{\square}{\Phi}$ (Figs. $9 b$ and $c$ ).

In this case the centre of the ventricular septal defect, which lay below the aortic valve (Fig. $9 d$ ), was not in line with the centre of the aortic sinuso

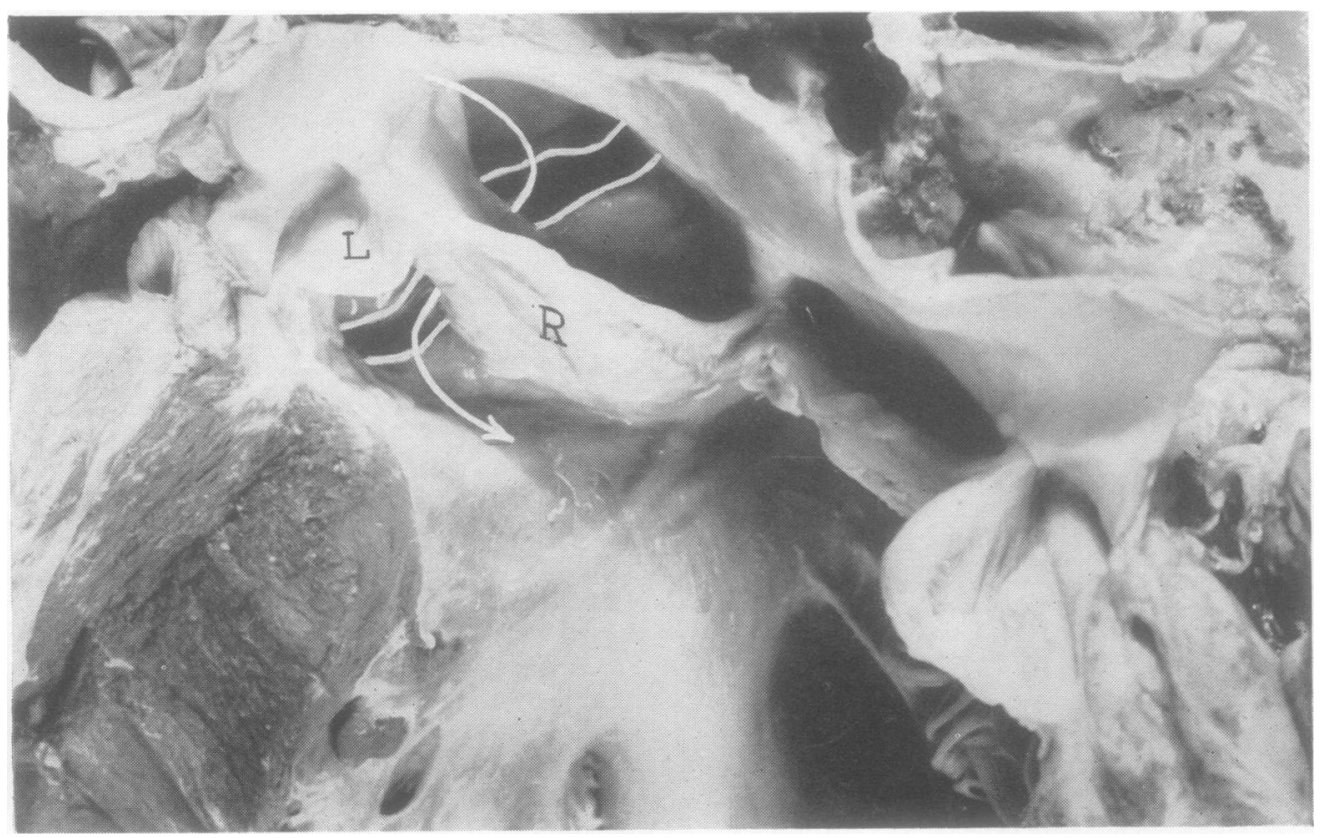

Fig. 8b 


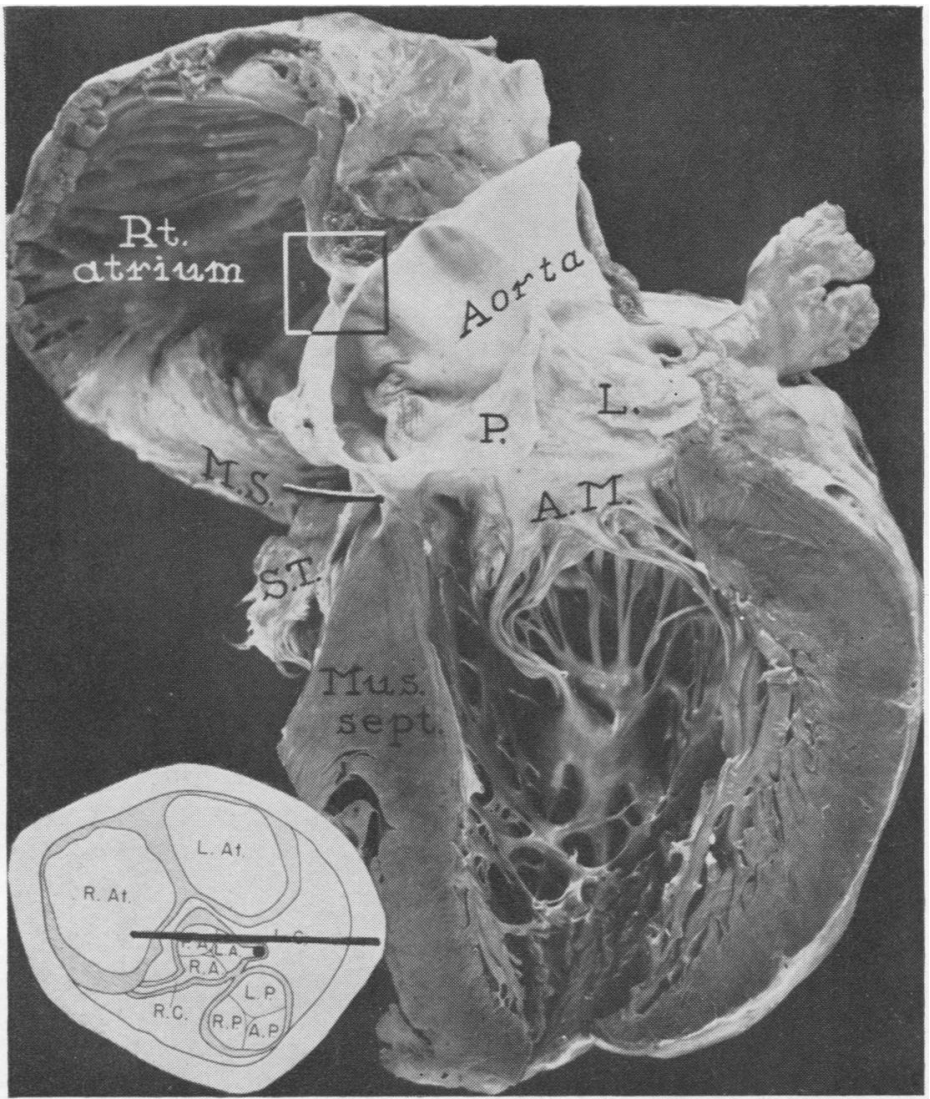

aneurysm, the subvalvular defect being in a more anterior position. From the right ventricular aspect the subvalvular defect opened into the right ventricle posteroinferior to the crista supraventricularis and just in front of the membranous portion of the ventricular septum.

In this case there was also an accessory orifice of the tricuspid valve through which the stream constituting the shunt from the left ventricle could enter the right atrium when the main tricuspid orifice was closed (Fig. 9b).

The findings of this case are duplicated in that of Herson and Symons (1946). The case of the latter authors had in addition a second aortic sinus aneurysm which involved the right aortic sinus and was not ruptured.

\section{COMMENT}

This communication does not primarily concern itself with those deficiencies between the aortic media and the heart that develop as a result of infection. At the same time it is recognized that

Fig. 9a

Fig. 9.-From a 28-year-old man with an aneurysm of the posterior aortic sinus and a ventricular septal defect associated with a double orifice of the tricuspid valve. a.-Sagittal section of heart through central portion of posterior aortic sinus (plane of section indicated in inset). There is a large aneurysm of the posterior aortic sinus, which protrudes into the right atrium through its septal wall. (Area in rectangle shown as photomicrograph in c.) b.-Close-up view of right atrial aspect of aneurysm of posterior aortic sinus. Two openings are present in the aneurysm, which lies just above the septal leaflet of the tricuspid valve. Probe is in the accessory orifice of the tricuspid valve, through which blood that shunted through the coexisting ventricular septal defect could enter the right atrium.

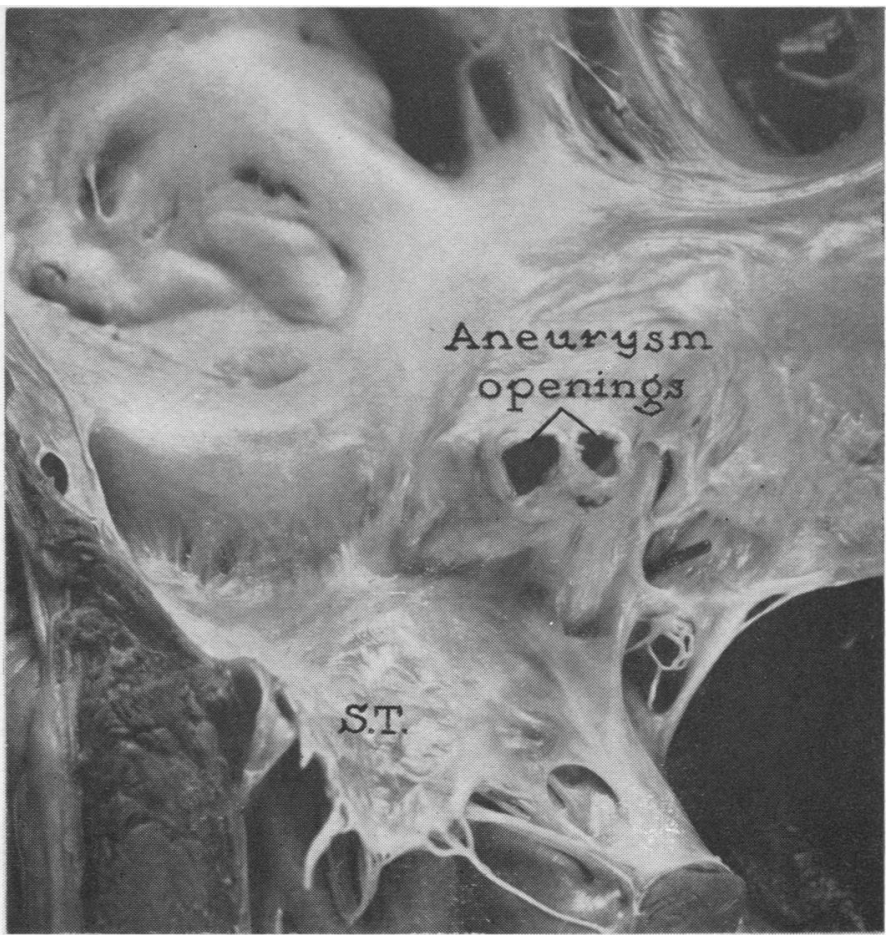

Fig. 9b 


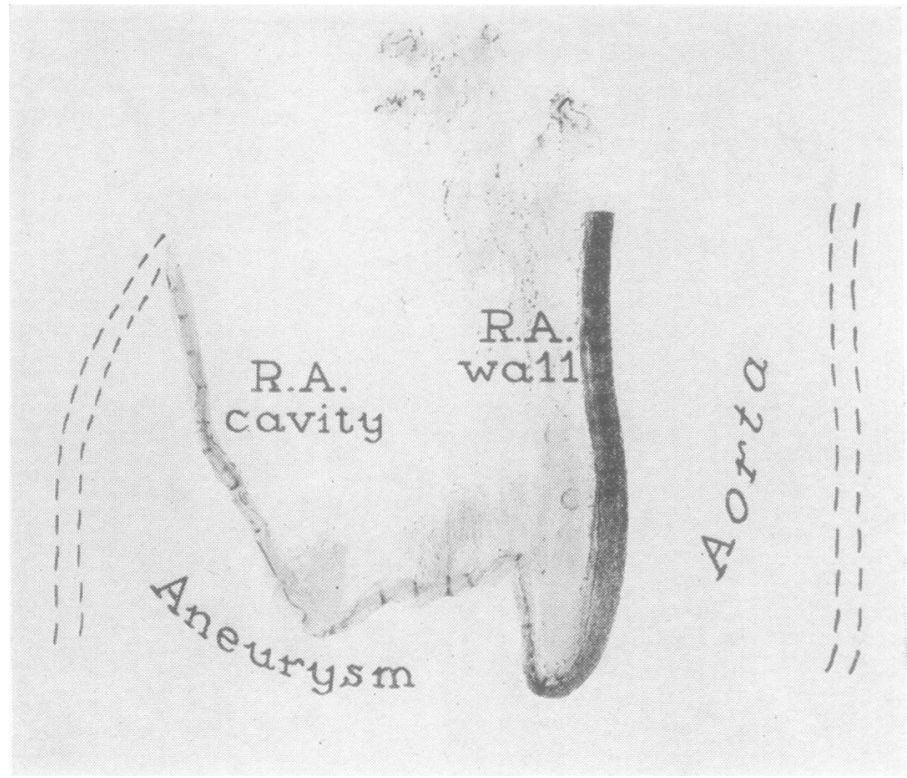

FIG. 9c.-Low-power photomicrograph through upper edge of aneurysm and right atrium. There is an abrupt interruption of the aorta, at which level the aneurysm originates. d.-Left ventricle and aortic valve. A small ventricular septal defect (point of arrow) lies just beneath the aortic valve at the level of the commissure between the right (R.) and the posterior (P.) aortic leaflet. The aneurysm of the posterior aortic sinus is hidden from view by the posterior aortic cusp.

Fig. 9c

lesions in the same anatomico-pathological sphere might complicate bacterial endocarditis. Here, too, the peculiarities of the lesion would depend on the particular areas related to the aortic valve that were involved.

We stress that, in cases with ruptured aneurysms of an aortic sinus and in cases of ventricular septal defect, bacterial endocarditis may occur as a complication (Jones and Langley, 1949; Abbott, 1919). At the same time, it is realized that in a heart without a spontaneous defect the destructive features of bacterial endocarditis may lead to a defect of continuity between the aorta and the heart. These facts make it obvious that, in a case with a defect in this area and with bacterial endocarditis also existing, it may be difficult or impossible to decide which was the primary lesion and which the complicating one.

In the cases that were discussed, the defects that lay inferior to the aortic valve and that appeared as interventricular communications are all considered congenital defects and are generally classified as ventricular septal defects.

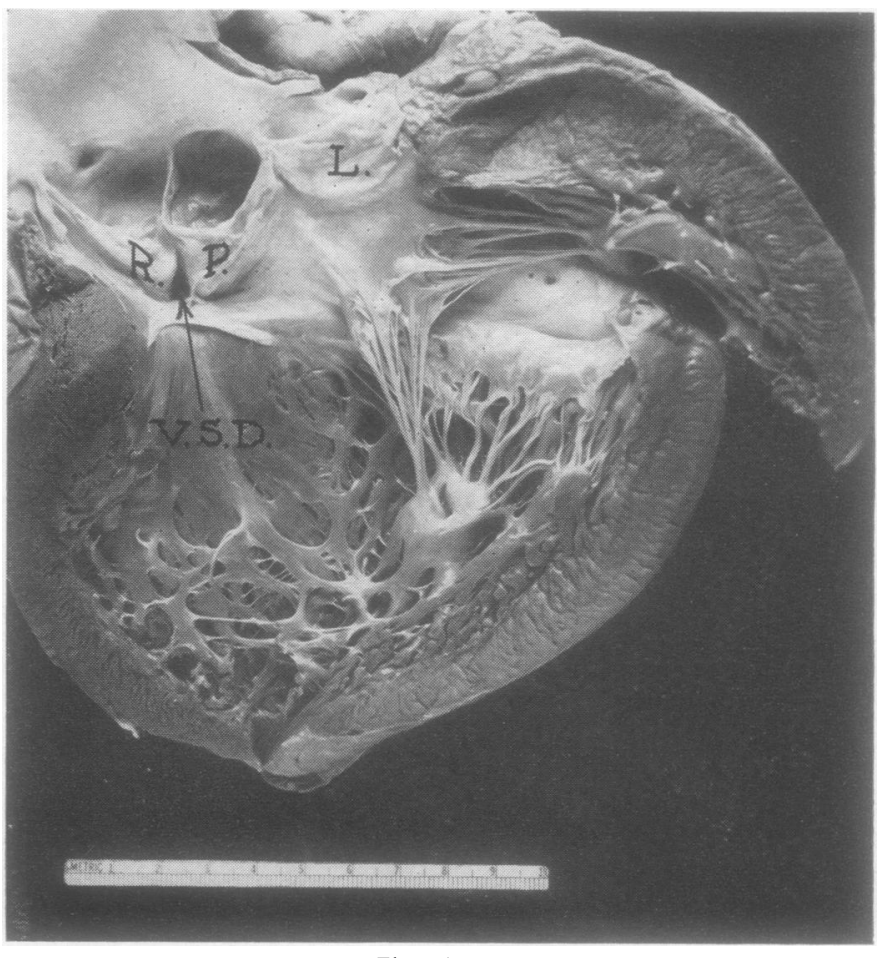

Fig. 9d 
We have indicated that such defects represent a deficiency of the normal continuity between the aorta and the heart. This deficiency may represent either failure of union or secondary separation.

It is not known whether those deficiencies that occur between the aorta above the valve and the heart develop before or after birth. It is possible that a congenital weakness in these areas is responsible for post-natal separation. Venning (1951) credited Dr. C. V. Harrison with the suggestion that the fundamental defect in aortic sinus aneurysm should be regarded as a defect of development of elastic tissue at the base of the aorta. This view supports the concept of "late"-either before or after birth-separation in this region.

Absence of inflammatory reaction in relation to aneurysms of the aortic sinus, as observed by Kawasaki and Benenson (1946), by Maynard and Thompson (1948), and by ourselves, supports a congenital basis for the genesis of these aneurysms.

Whatever the time of appearance of the aortic sinus aneurysm, when it ruptures it does so almost invariably in post-natal, usually adult, life.

The observations cited suggest that proper repair of deficiencies of the types described requires that the aortic wall be reunited, either directly or indirectly by a prosthesis, with the heart.

Mere closure of an aortic aneurysm, without reuniting the aortic media with the heart, fails to overcome the basic lesion.

\section{SUMmaRY}

A series of lesions representing separation between the aortic wall and the heart are described. The gross pathological nature of the lesion depends on which of the three aortic cusps it is related to. It also depends on whether the deficiency occurs above or below the aortic valve.

Those deficiencies that occur above the valve are aortic sinus aneurysms, while most of those that lie below the valve usually represent ventricular septal defect. Rarely, the picture of aneurysm of the left ventricular base results.

Two or more lesions may coexist.

The anatomical relations of the normal three aortic cusps and sinuses are reviewed.

\section{REFERENCES}

Abbott, M. E. (1919). In Contributions to Medical and Biological Research, dedicated to Sir William Osler, vol. 2, p. 899. Hoeber, New York.

Barnard, P. J., and Brink, A. J. (1956). Brit. Heart J., 18, 309.

Becu, L. M., Fontana, R. S., DuShane, J. W., Kirklin, J. W., Burchell, H. B., and Edwards, J. E. (1956). Circulation, 14, 349.

Burchell, H. B., and Edwards, J. E. (1951). Proc. Mayo Clin., 26, 336. Edwards, J. E., Burchell, H. B., and Christensen, N. A. (1956). Ibid., 31, 407, 464.

Gross, L., and Kugel, M. A. (1931). Amer. J. Path., 7, 445.

Herson, R. N.. and Symons, M. (1946). Brit. Heart J, 8, 125.

Herson, R. N.. and Symons, M. (1946). Brit. Heart J.,
Higgins, A. R. (1934). Nav. med. Bull. (Wash.), 32, 47.

Jones, A. M., and Langley, F. A. (1949). Brit. Heart J., 11, 325.

Kawasaki, I. A., and Benenson, A. S. (1946). Ann. intern. Med., 25, 150.

Macleod, A. (1944). Brit. Heart J., $6,194$.

Maynard, R. M., and Thompson, C.W. (1948). Arch. Path. (Chicago), 45,65 .

Micks, R. H. (1940). Brit. Heart J., $2,63$.

Morgan, E. H., and Burchell, H. B.(1950). Proc. Mayo Clin., 25, 69.

Oram, S., and East, T. (1955). Brit. Heart J., 17, 541.

Orbison, J. L., and Mostofi, F. K. (1956), Amer. Heart J., 51, 636.

Ostrum, H. W., Robinson, B. D., Nichols, C. F., and Widmann, B. P. (1938). Amer. J. Roentgenol., 40, 828.

Raman, T. K., and Menon, T. B. (1949). Indian Heart J., 1, 1.

Venning, G. R. (1951). Amer. Heart J., 42, 57.

Warthen, R. O. (1949). Ibid., 37, 975. 